\title{
Identification of shared and unique gene families associated with oral clefts
}

\author{
Noriko Funato and Masataka Nakamura
}

Oral clefts, the most frequent congenital birth defects in humans, are multifactorial disorders caused by genetic and environmental factors. Epidemiological studies point to different etiologies underlying the oral cleft phenotypes, cleft lip (CL), $\mathrm{CL}$ and/or palate (CL/P) and cleft palate (CP). More than 350 genes have syndromic and/or nonsyndromic oral cleft associations in humans. Although genes related to genetic disorders associated with oral cleft phenotypes are known, a gap between detecting these associations and interpretation of their biological importance has remained. Here, using a gene ontology analysis approach, we grouped these candidate genes on the basis of different functional categories to gain insight into the genetic etiology of oral clefts. We identified different genetic profiles and found correlations between the functions of gene products and oral cleft phenotypes. Our results indicate inherent differences in the genetic etiologies that underlie oral cleft phenotypes and support epidemiological evidence that genes associated with CL/P are both developmentally and genetically different from CP only, incomplete CP, and submucous CP. The epidemiological differences among cleft phenotypes may reflect differences in the underlying genetic causes. Understanding the different causative etiologies of oral clefts is important as it may lead to improvements in diagnosis, counseling, and prevention.

International Journal of Oral Science (2017) 9, 104-109; doi:10.1038/ijos.2016.56; published online 20 January 2017

Keywords: cleft palate; epidemiology; gene ontology; mutations; soft palate; syndrome

\section{INTRODUCTION}

Oral clefts are common multifactorial birth defects presenting with a wide range of abnormalities in the upper lip, the primary palate, and the secondary palate, and include cleft lip (CL), cleft palate (CP), CL and/or palate $(\mathrm{CL} / \mathrm{P})$, incomplete $\mathrm{CP}$, and submucous $\mathrm{CP} \cdot{ }^{1-2}$ Because the secondary palate consists of both a bone-lined hard palate and a bone-free soft palate, incomplete CP includes hard-palate cleft, softpalate cleft, and bifid uvula. The mildest forms of CP are defects of the soft palate only (soft-palate cleft) or the uvula only (bifid uvula). Oral clefts may be nonsyndromic or manifest as a clinical phenotype within syndromes. They can be caused by different etiological factors such as single gene mutations, chromosomal aberrations, and specific environmental agents as well as by interactions between genetic and environmental influences. ${ }^{3-4}$ Concordance rates for CL, CL/P, and $\mathrm{CP}$ are higher in monozygotic twins than in dizygotic twins, ${ }^{5}$ which indicates significant, but not exclusive, genetic contributions. Epidemiological studies indicate that oral cleft phenotypes may have different underlying etiologies. For instance, isolated $\mathrm{CP}$ and $\mathrm{CL} / \mathrm{P}$ seldom occur in the same family. ${ }^{3}$ Siblings of patients with CL/P have an increased frequency of $\mathrm{CL} / \mathrm{P}$ but not of isolated $\mathrm{CP}$, while siblings of patients with isolated $\mathrm{CP}$ have an increased frequency of isolated $\mathrm{CP}$ but not of CL/P. ${ }^{3}$ Moreover, CL/P and CP display different sex ratios and prevalence among oral cleft phenotypes. The recurrence risk of
$\mathrm{CP}$ among siblings is higher in females than in males whereas the reverse is true for $\mathrm{CL} / \mathrm{P} .^{3-6}$ Gaining insight into the different causative etiologies of oral clefts is important as it may lead to improved diagnosis, counseling, and preventive health treatments.

Oral clefts in humans are associated with a large number of genetic diseases/syndromes, ${ }^{7}$ and findings from studies using genetically engineered mice with oral cleft have improved our understanding of palatogenesis. ${ }^{8-9}$ As a result, many genetic mutations associated with human and mouse oral clefts have been identified and molecular functions have been elucidated. Since the identification and functional classification of disease-causing genes can reveal general biological mechanisms underlying human diseases and disorders, ${ }^{10}$ investigating the functional annotation of candidate genes associated with oral clefts would aid in a better understanding not only of the biological basis of these phenotypically variable and complex group of conditions but also of their underlying genetic causes.

\section{MATERIALS AND METHODS}

Genes associated with human oral cleft phenotypes

Online Mendelian Inheritance in Man (OMIM) (http://omim.org) ${ }^{11}$ is a comprehensive, well-established database of human genes and genetic disorders integrating genetic information with clinical phenotypes and diseases in humans. Similarly, the GATACA database 
(https://gataca.cchmc.org/gataca/) provides links between genes and different diseases or phenotypes using cross mapping to identify genetic overlap between different biological elements, functions, or processes. In our evaluation of the genetic basis of human palatogenesis, we first investigated congenital disorders or syndromes associated with oral clefts and their candidate genes using OMIM and GATACA. The databases were searched using the terms "cleft lip and palate", "cleft lip/palate", "cleft lip and/or palate", "cleft lip", "cleft of upper lip", "cleft palate", "cleft secondary palate", "incomplete cleft palate", "submucosal cleft palate", "submucous cleft palate", "soft palate cleft", "cleft of the soft palate", "soft cleft palate", "cleft uvula", and "bifid uvula". The search was completed on 9 February 2016. In our identification of oral cleft phenotypes in humans, our primary search results were screened using the following exclusion criteria: (1) genes associated with oral clefts in mice with no evidence of association in humans; and (2) genes specifically associated with an absent uvula. The resulting list of genes associated with nonsyndromic and syndromic oral cleft phenotypes in humans was used for ontology analysis. Positive hits were further interrogated to identify oral cleft subphenotypes through review of either the Clinical Synopsis or articles cited in OMIM (Supplementary Table 1 and Supplementary References). OMIM and the NCBI Gene database (http://www.ncbi. nlm.nih.gov/gene) were used to identify the corresponding proteins and Entrez Gene ID of each gene.

\section{Gene ontology analysis}

For a better understanding of the genetic contributions underlying oral clefts, genes associated with oral cleft were further analyzed based on biological process, molecular function, and gene family using the Protein ANalysis THrough Evolutionary Relationships (PANTHER) a

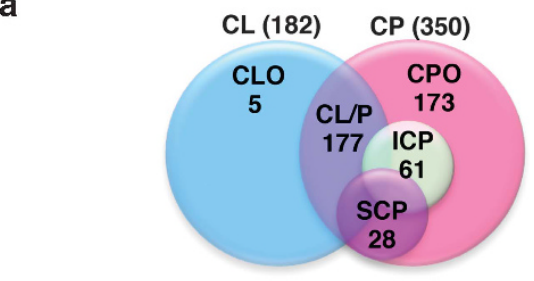

b

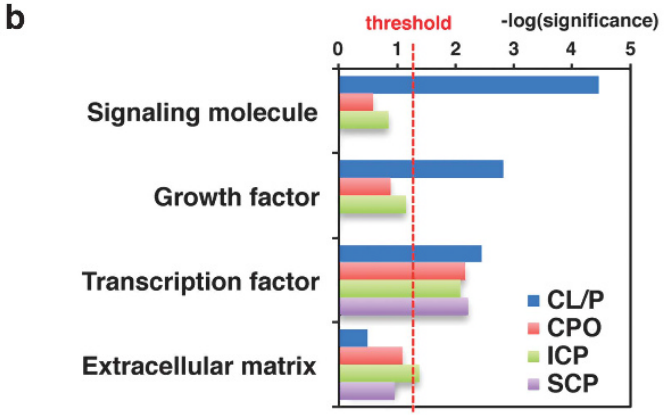

C

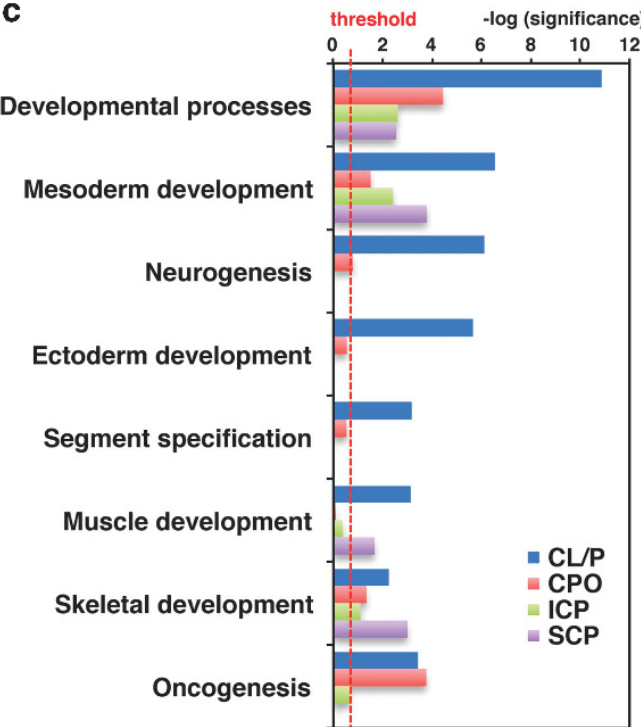

d

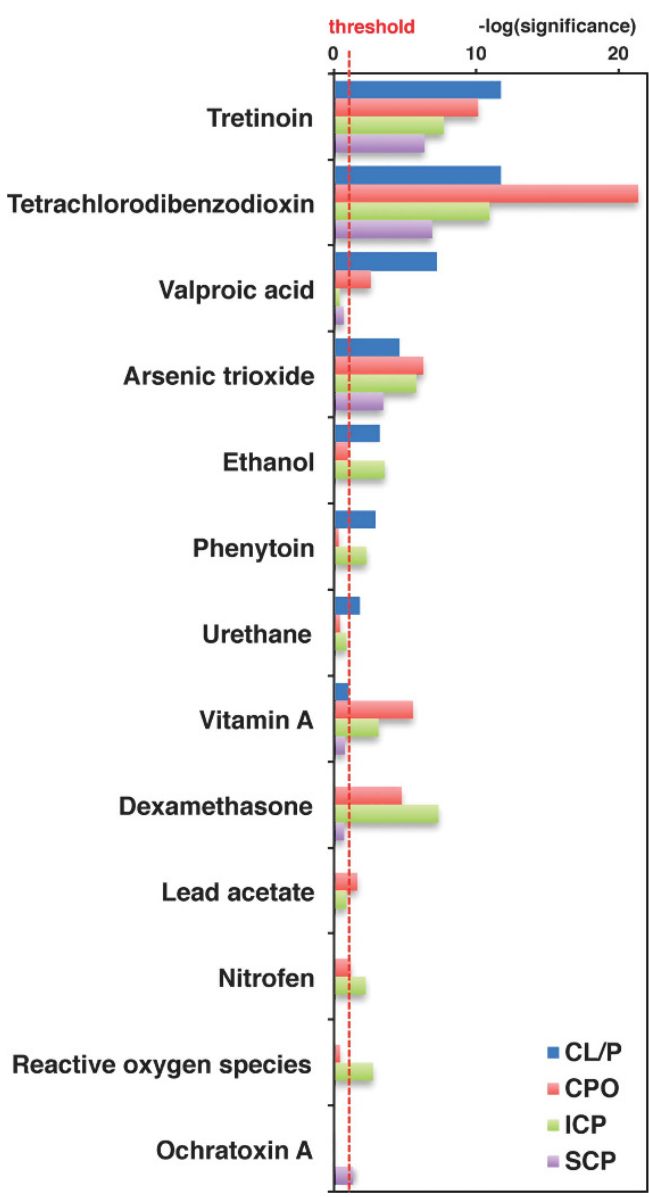

Figure 1 Gene profiles differ depending on cleft palate phenotype. (a) The overlap between human genes associated with cleft phenotypes is depicted in the Venn diagram. The numbers in each area represent the gene count for the particular section. (b-d) Gene ontology analysis of genes associated with human cleft palate phenotypes according to molecular function (b), biological process (c) and chemicals (d). Plotted is the $-\log (P$-value) with the threshold set to $1.3[\log (0.05)]$. CP, cleft palate; CL/P, cleft lip and/or palate; CPO, cleft palate only; ICP, incomplete cleft palate; SCP, submucous cleft palate; CL, cleft lip; CLO, cleft lip only. 
database (http://pantherdb.org). ${ }^{12}$ Briefly, Entrez Gene IDs were uploaded to identify unique and annotated genes for inclusion in the ontology analysis. The resulting gene lists were evaluated using tests for enrichment that identify functional classes in which the genes of a given class have values that are non-randomly selected from a genome-wide distribution of values. ${ }^{12}$ Statistically significant enrichment of the data set in a given process was determined using binomial testing with Bonferroni corrections for multiple testing as described previously. ${ }^{13}$ Only those classes demonstrating statistically significant $(P<0.05)$ enrichment were used for gene family analysis. Putative chemical-gene-disease interactions were identified using the Comparative Toxicogenomics Database (CTD) (http://ctdbase.org). ${ }^{14}$ For CTD analysis, derived nominal $P$-values were adjusted using the false discovery rate as described by Benjamini and Yekutieli. ${ }^{15}$ The CTD contains many classes with similar protein constituents. Therefore, the gene counts of those classes that were a complete subset of another were discarded.

\section{RESULTS}

Gene profiles differ depending on the oral cleft phenotype

As a result of our search using OMIM and GATACA (refer to Materials and Method section for a full list of search terms), we found over 350 candidate genes having one or more syndromic and/or nonsyndromic oral cleft annotations (Supplementary Table 1). Since phenotypic classification of human genes often yields important insights into gene function, ${ }^{16}$ we classified the identified genes based on their association with $\mathrm{CL} / \mathrm{P}, \mathrm{CP}$ only (CPO), incomplete $\mathrm{CP}$, and submucous $\mathrm{CP}$ as shown in Figure 1a.
To investigate whether gene profiles differ among oral cleft phenotypes, we performed a gene ontology analysis first comparing candidate genes using the PANTHER database (Figure $1 \mathrm{~b}$ and $1 \mathrm{c}$ and Table 1-3). Based on studies that investigated expression patterns and phenotypes in mutant mice, homeobox transcription factors have roles in the patterning of the upper and lower jaws. ${ }^{17-18}$ We found that when genes were analyzed according to molecular function, those found in the transcription factor category, especially those genes that contain a homeobox transcription domain, were enriched in all oral cleft phenotypes (Figure 1b, Table 1, family \#1 in Table 3). We also found that genes associated with signaling molecules $(P=0.000035)$ and growth factor $(P=0.0015)$ were significantly enriched in $C L / P$, and genes associated with the extracellular matrix were significantly enriched in incomplete CP $(P=0.042)$ (Figure $1 \mathrm{~b}$ and Table 1). When genes were analyzed according to biological process, neurogenesis ( $P=0.00000076)$, ectoderm development $(P=0.0000021)$, and segment specification $(P=0.00066)$ were enriched in only $\mathrm{CL} / \mathrm{P}$ (Figure $1 \mathrm{c}$ and Table 2). In submucous $\mathrm{CP}$, we found that muscle development $(P=0.0021)$ and skeletal development $(P=0.00099)$ were enriched (Figure 1c and Table 2). Developmental process and mesoderm development were significantly enriched in all oral cleft phenotypes (Figure 1c).

We next investigated possible chemical-gene-disease interactions using the CTD to investigate the mechanisms underlying environmentally influenced oral clefts. We found that the enrichment distribution of chemicals was also different among cleft phenotypes (Figure 1d). Tretinoin (the carboxylic acid form of vitamin A), tetrachlorodibenzodioxin (also known as Dioxin), and arsenic trioxide

Table 1 Classification of candidate genes associated with human oral cleft phenotypes according to molecular function

\begin{tabular}{|c|c|c|c|c|}
\hline Molecular function & Cleft type & $\%$ & $P$-value & Genes \\
\hline \multirow[t]{4}{*}{ Signaling molecule } & CL/P & 12.4 & $3.5 \times 10^{-5}$ & $\begin{array}{l}\text { ANK1, BMP4, EFNB1, FGF1*, FGF10, FGF17, FGF19*, FGF2*, FGF8, GRIP1, IL1RN, IL1B, JAG2*, } \\
N^{*} G^{*}, \text { PDGFC }{ }^{*}, \text { SEMA3E, SHH, SPRY2*, TGFA, WNT3, WNT5A, WNT7A }\end{array}$ \\
\hline & $\mathrm{CPO}$ & 6.4 & $2.6 \times 10^{-1}$ & STAMBP, BMP2, CRLF1, EDN1, GNRH1, GDF1, GDF6, PLCB4, SPRY4, TGFB2, TGFB3 \\
\hline & ICP & 9.8 & $1.4 \times 10^{-1}$ & BMP4, CRLF1, EDN1, GDF1, TGFB2, TGFB3 \\
\hline & SCP & 0.0 & $\mathrm{~N} / \mathrm{A}$ & $\mathrm{N} / \mathrm{A}$ \\
\hline \multirow[t]{4}{*}{ Growth factor } & CL/P & 4.0 & $1.5 \times 10^{-3}$ & $F G F 1^{*}, F G F 10, F G F 17, F G F 19^{*}, F G F 2^{*}, F G F 8, P D G F C^{*}$ \\
\hline & CPO & 2.3 & $1.3 \times 10^{-1}$ & GDF1, GDF6, TGFB2, TGFB3 \\
\hline & $\mathrm{ICP}$ & 4.9 & $7.1 \times 10^{-2}$ & GDF1, TGFB2, TGFB3 \\
\hline & SCP & 0.0 & N/A & $\mathrm{N} / \mathrm{A}$ \\
\hline \multirow[t]{4}{*}{ Transcription factor } & CL/P & 18.6 & $3.6 \times 10^{-3}$ & 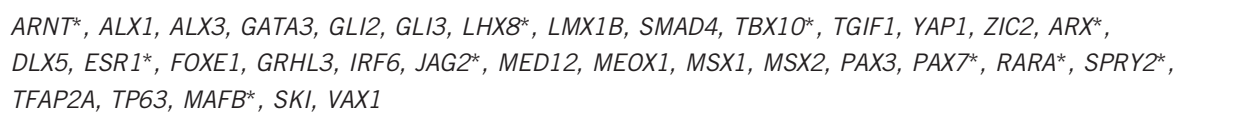 \\
\hline & $\mathrm{CPO}$ & 18.5 & $6.8 \times 10^{-3}$ & $\begin{array}{l}\text { ALX4, CTCF, FEZF1, GATA6, KAT6B, MKX*, NKX2-5, NKX2-6, SATB2, SMAD3, SOX2, SOX9, TBX1, } \\
\text { TBX15, TBX22, TBX4, WT1, ZIC3, FOXC2, HOXA2, OTX2, PRRX1, PITX1, PQBP1, RB1, RAI1, RARB, RUNX2, SRY, } \\
\text { SPRY4, TWIST1, ZEB2 }\end{array}$ \\
\hline & $\mathrm{ICP}$ & 9.8 & $8.3 \times 10^{-3}$ & GATA6, GL13, NKX2-5, NKX2-6, SATB2, SMAD3, TBX1, TBX22, DLX5, IRF6, TP63 \\
\hline & SCP & 32.1 & $6.1 \times 10^{-3}$ & GATA6, NKX2-5, NKX2-6, TBX1, TBX22, DLX5, MED12, RUNX2, ZEB2 \\
\hline \multirow[t]{4}{*}{ Transferase } & CL/P & 6.2 & $3.6 \times 10^{-1}$ & MTR, NAT1*, NAT2* NEK1, WHSC1, B3GLCT, ESCO2, GSTT1* LARGE, POMT1, POMT2 \\
\hline & CPO & 8.7 & $5.0 \times 10^{-2}$ & $\begin{array}{l}\text { GMPPB, KAT6B, B3GALT6, ALG3, B3GAT3, CHST14, COMT*, CHSY1, PSAT1, HS6ST1, KMT2D, } \\
\text { NSD1, PTDSS1, POLR1D, XYLT1 }\end{array}$ \\
\hline & ICP & 3.3 & $9.6 \times 10^{-1}$ & $A L G 3, C^{*}$ \\
\hline & SCP & 0.0 & N/A & $\mathrm{N} / \mathrm{A}$ \\
\hline \multirow[t]{4}{*}{ Extracellular matrix } & $\mathrm{CL} / \mathrm{P}$ & 3.4 & $3.2 \times 10^{-1}$ & MKS1, COL8A1*, FLRT3, MMP9* NTN1*, NOG* \\
\hline & СPO & 4.6 & $8.1 \times 10^{-2}$ & BMPER, COL2A1, COL9A2, COL11A1, COL11A2, GPC3, MEGF10, TNXB \\
\hline & ICP & 8.2 & $4.2 \times 10^{-2}$ & COL2A1, COL11A1, COL11A2, GPC3, TNXB \\
\hline & SCP & 10.7 & $1.1 \times 10^{-1}$ & COL11A1, COL11A2, GPC3 \\
\hline
\end{tabular}

CL/P, cleft lip and/or palate; CPO, cleft palate only; ICP, incomplete cleft palate; SCP, submucous cleft palate; N/A, not applicable; \%, involved genes/total genes $\times 100$; $P$-value, probabilities were adjusted for multiple comparisons across all PANTHER molecular functions using Bonferroni correction.

${ }^{*}$ Genes associated with nonsyndromic oral clefts. 
(an anti-cancer chemotherapy drug) were significantly enriched in all oral cleft phenotypes (Figure 1d). Valproic acid, a medication primarily used to treat epilepsy and bipolar disorder, was significantly enriched in CL/P $(P=0.00000006144)$ and $\mathrm{CPO}(P=0.002719)$, but not in incomplete CP and submucous CP (Figure 1d). In addition, we found that ethanol and phenytoin (an anti-seizure medication) were both enriched in $\mathrm{CL} / \mathrm{P}$ and incomplete $\mathrm{CP}$ (Figure 1d), whereas vitamin $\mathrm{A}$ and dexamethasone (a corticosteroid) were both enriched in $\mathrm{CPO}$ and incomplete $\mathrm{CP}$ (Figure 1d). The herbicide nitrofen and reactive oxygen species were significantly enriched in incomplete $\mathrm{CP}$, whereas ochratoxin A, which is a mycotoxin produced by Aspergillus ochraceus, was enriched specifically in submucous CP (Figure 1d).

We also analyzed genes according to gene family. Interestingly, gene products involved in the TGF- $\beta$ signaling pathway (family \#4 in Table 3) were enriched in CPO $(P=0.00024)$ and incomplete CP $(P=0.00019)$ whereas genes involved in the fibroblast growth factor (FGF) family were only enriched in $\mathrm{CL} / \mathrm{P}(P=0.0000032)$ (family \#5 in Table 3). In addition, we found that all three of the T-box protein, collagen-a chain protein, and TGF- $\beta$ families were associated with $\mathrm{CPO}$ and incomplete CP (families \#2-4 in Table 3).

Table 2 Classification of candidate genes associated with human oral cleft phenotypes according to biological process

\begin{tabular}{|c|c|c|c|c|}
\hline Biological process & Cleft type & $\%$ & $P$-value & Genes \\
\hline \multirow[t]{4}{*}{$\begin{array}{l}\text { Developmental } \\
\text { processes }\end{array}$} & $\mathrm{CL} / \mathrm{P}$ & 31.6 & $1.3 \times 10^{-11}$ & 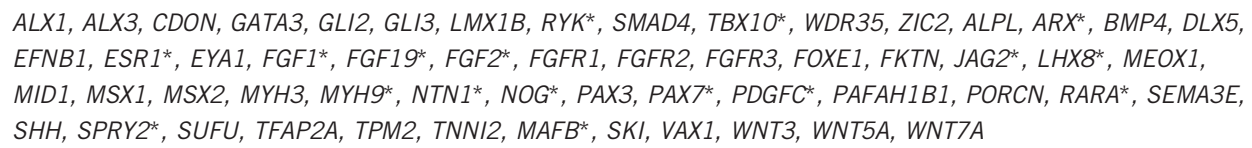 \\
\hline & $\mathrm{CPO}$ & 24.3 & $3.6 \times 10^{-5}$ & $\begin{array}{l}\text { ALX4, BMPER, GATA6, L1CAM, KAT6B, NKX2-5, NKX2-6, SMAD3, TBX1, TBX15, TBX22, TBX4, TCOF1, WT1, ZIC3, } \\
\text { BMP2, BUB1B, COL9A2, COL11A1, COL11A2, FLVCR2, FOXC2, GNRH1, GNRHR, GDF1, HOXA2, LRP4, MEGF10, } \\
\text { OTX2, PRRX1, PDGFRA*, PTPN11, RB1, RARB, RUNX2, SPRY4, SMC1A, TNXB, TGFB2, TGFB3, TGFBR2, TWIST1 }\end{array}$ \\
\hline & $\mathrm{ICP}$ & 27.9 & $2.5 \times 10^{-3}$ & $\begin{array}{l}\text { GATA6, GL13, NKX2-5, NKX2-6, SMAD3, TBX1, TBX22, BMP4, COL11A1, COL11A2, EYA1, FGFR2, GDF1, TNXB, } \\
\text { TGFB2, TGFB3, TGFBR2 }\end{array}$ \\
\hline & SCP & 35.7 & $2.8 \times 10^{-3}$ & GATA6, NKX2-5, NKX2-6, TBX1, TBX22, COL11A1, COL11A2, DLX5, FGFR1, RUNX2 \\
\hline \multirow[t]{4}{*}{$\begin{array}{l}\text { Mesoderm } \\
\text { development }\end{array}$} & $\mathrm{CL} / \mathrm{P}$ & 12.4 & $2.9 \times 10^{-7}$ & $\begin{array}{l}\text { ALX1, CDON, GATA3, GLI3, ALPL, BMP4, DLX5, FGF1* FGF2*, FOXE1, FKTN, MEOX1, MSX1, MSX2, MYH3, MYH9*, } \\
\text { NOG }^{*} \text {, SPRY2*, SUFU, TPM2, TNNI2, SKI }\end{array}$ \\
\hline & CPO & 6.9 & $3.1 \times 10^{-2}$ & NKX2-5, NKX2-6, TBX22, TBX4, BMP2, COL9A2, COL11A1, COL11A2, FOXC2, GDF1, RUNX2, SPRY4 \\
\hline & $\mathrm{ICP}$ & 13.1 & $3.8 \times 10^{-3}$ & GL13, NKX2-5, NKX2-6, TBX22, BMP4, COL11A1, COL11A2, GDF1 \\
\hline & SCP & 25.0 & $1.6 \times 10^{-4}$ & NKX2-5, NKX2-6, TBX22, COL11A1, COL11A2, DLX5, RUNX2 \\
\hline \multirow[t]{4}{*}{ Neurogenesis } & $\mathrm{CL} / \mathrm{P}$ & 12.4 & $7.6 \times 10^{-7}$ & 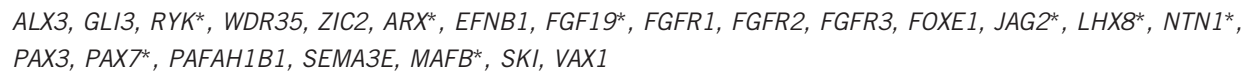 \\
\hline & CPO & 5.8 & $1.6 \times 10^{-1}$ & ALX4, L1CAM, TCOF1, ZIC3, FOXC2, HOXA2, MEGF10, OTX2, PRRX1, TNXB \\
\hline & $\mathrm{ICP}$ & 4.9 & $6.4 \times 10^{-1}$ & GLI3, FGFR2, TNXB \\
\hline & SCP & 3.6 & 1.0 & FGFR1 \\
\hline \multirow[t]{4}{*}{$\begin{array}{l}\text { Ectoderm } \\
\text { development }\end{array}$} & $\mathrm{CL} / \mathrm{P}$ & 13.0 & $2.1 \times 10^{-6}$ & 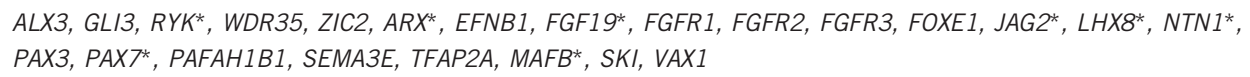 \\
\hline & $\mathrm{CPO}$ & 5.8 & $2.8 \times 10^{-1}$ & ALX4, LICAM, TCOF1, ZIC3, FOXC2, HOXA2, MEGF10, OTX2, PRRX1, TNXB \\
\hline & $\mathrm{ICP}$ & 4.9 & $7.2 \times 10^{-1}$ & GLI3, FGFR2, TNXB \\
\hline & SCP & 3.6 & 1.0 & FGFR1 \\
\hline \multirow{4}{*}{$\begin{array}{l}\text { Segment } \\
\text { specification }\end{array}$} & $\mathrm{CL} / \mathrm{P}$ & 4.0 & $6.6 \times 10^{-4}$ & $A L X 3, A R X^{\star}, D L X 5, F O X E 1, P A X 3, P A X 7^{*}, P O R C N$ \\
\hline & CPO & 1.7 & $2.9 \times 10^{-1}$ & ALX4, FOXC2, HOXA2 \\
\hline & $\mathrm{ICP}$ & 0.0 & $\mathrm{~N} / \mathrm{A}$ & $\mathrm{N} / \mathrm{A}$ \\
\hline & SCP & 3.6 & 1.0 & $D L X 5$ \\
\hline \multirow{4}{*}{$\begin{array}{l}\text { Skeletal } \\
\text { development }\end{array}$} & $\mathrm{CL} / \mathrm{P}$ & 4.5 & $3.8 \times 10^{-4}$ & $A L X 1, A L P L, B M P 4, D L X 5, M S X 1, M S X 2, N O G^{*}, S U F U$ \\
\hline & CPO & 2.9 & $4.5 \times 10^{-2}$ & NKX2-5, NKX2-6, BMP2, COL9A2, RUNX2 \\
\hline & $\mathrm{ICP}$ & 4.9 & $7.9 \times 10^{-2}$ & $N K X 2-5, N K X 2-6, B M P 4$ \\
\hline & SCP & 14.3 & $9.9 \times 10^{-4}$ & NKX2-5, NKX2-6, DLX5, RUNX2 \\
\hline \multirow{4}{*}{$\begin{array}{l}\text { Muscle } \\
\text { development }\end{array}$} & $\mathrm{CL} / \mathrm{P}$ & 4.5 & $7.3 \times 10^{-4}$ & CDON, DLX5, FKTN, MYH3, MYH9*, TPM2, TNNI2, SKI \\
\hline & CPO & 1.2 & $7.8 \times 10^{-1}$ & $N K X 2-5, N K X 2-6$ \\
\hline & $\mathrm{ICP}$ & 3.3 & $4.1 \times 10^{-1}$ & $N K X 2-5, N K X 2-6$ \\
\hline & SCP & 10.7 & $2.1 \times 10^{-2}$ & $N K X 2-5, N K X 2-6, D L X 5$ \\
\hline \multirow[t]{4}{*}{ Oncogenesis } & $\mathrm{CL} / \mathrm{P}$ & 6.8 & $5.6 \times 10^{-3}$ & SMAD4, FGFR1, FGFR2, FGFR3, IRF6, RARA*, SHH, SUFU, TFAP2A, TP63, KRAS, SKI \\
\hline & CPO & 8.7 & $1.7 \times 10^{-4}$ & BRIP1, SMAD3, WT1, BUB1B, CDKN1C, LOXL3, NRAS, PTEN, PDGFRA*, RB1, RARB, RUNX2, ST5*, HRAS, BRAF \\
\hline & $\mathrm{ICP}$ & 6.6 & $2.1 \times 10^{-1}$ & SMAD3, FGFR2, IRF6, TP63 \\
\hline & SCP & 10.7 & $1.5 \times 10^{-1}$ & FGFR1, RUNX2, BRAF \\
\hline
\end{tabular}

CL/P, cleft lip and/or palate; CPO, cleft palate only; ICP, incomplete cleft palate; SCP, submucous cleft palate; N/A, not applicable; \%, involved genes/total genes $\times 100$; $P$-value, probabilities were adjusted for multiple comparisons across all PANTHER molecular functions using Bonferroni correction.

* Genes associated with nonsyndromic oral clefts. 
Table 3 Classification of candidate genes associated with human oral cleft phenotypes according to gene family

\begin{tabular}{|c|c|c|c|c|c|c|}
\hline & \# & Domain name & $\begin{array}{l}\text { Cleft } \\
\text { type }\end{array}$ & $\%$ & P-value & Genes \\
\hline \multirow[t]{3}{*}{ CL/P, CPO and SCP } & 1 & Homeobox protein & $\mathrm{CL} / \mathrm{P}$ & 8.7 & $3.1 \times 10^{-5}$ & $\begin{array}{l}\text { ALX1, ALX3, ARX } X^{\star}, D L X 5, M S X 1, M S X 2, P A X 3, P A X 7^{\star}, V A X 1, L H X 8^{\star}, \\
\text { MEOX1, TGIF1, LMX1B, SIX3, }\end{array}$ \\
\hline & & & $\mathrm{CPO}$ & 5.5 & $4.9 \times 10^{-3}$ & $\begin{array}{l}\text { ALX4, HOXA2, MKX }, \text { NKX2-5, NKX2-6, OTX2, PITX1, PRRX1, SATB2, } \\
\text { SIX3, ZEB2 }\end{array}$ \\
\hline & & & SCP & 13.4 & $2.6 \times 10^{-2}$ & $D L X 5, N K X 2-5, N K X 2-6, S I X 3, Z E B 2$ \\
\hline & & & SCP & 7.1 & $2.5 \times 10^{-2}$ & $T B X 1, T B X 22$ \\
\hline \multirow[t]{4}{*}{ CPO and ICP } & 3 & Collagen alpha chain & $\mathrm{CPO}$ & 2.3 & $5.0 \times 10^{-2}$ & COL2A1, COL9A2, COL11A1, COL11A2 \\
\hline & & & $\mathrm{ICP}$ & 4.9 & $3.7 \times 10^{-2}$ & COL2A1, COL11A1, COL11A2 \\
\hline & 4 & TGF- $\beta$ family & $\mathrm{CPO}$ & 2.9 & $2.4 \times 10^{-4}$ & GDF6, GDF1, TGFB2, TGFB3, BMP2 \\
\hline & & & $\mathrm{ICP}$ & 6.6 & $1.9 \times 10^{-4}$ & GDF1, TGFB2, TGFB3, BMP4 \\
\hline \multirow[t]{7}{*}{ CL/P only } & 5 & Heparin-binding FGF family member & $\mathrm{CL} / \mathrm{P}$ & 3.4 & $3.2 \times 10^{-6}$ & FGF17, FGF1 ${ }^{*}$, FGF2 $^{*}$, FGF8, FGF10, FGF19* \\
\hline & 10 & Wnt related & $\mathrm{CL} / \mathrm{P}$ & 1.7 & $1.4 \times 10^{-2}$ & WNT3, WNT5A, WNT7A \\
\hline & 11 & $\mathrm{~N}$-hydroxyarylamine o-acetyltransferase & $\mathrm{CL} / \mathrm{P}$ & 1.1 & $1.9 \times 10^{-2}$ & $N A T 1^{*}, N A T 2^{*}$ \\
\hline & 12 & IFT140/172-related & $\mathrm{CL} / \mathrm{P}$ & 1.1 & $1.9 \times 10^{-2}$ & IFT140, IFT172 \\
\hline & 13 & $\begin{array}{l}\text { Dolichyl-phosphate-mannose-protein } \\
\text { mannosyltransferase }\end{array}$ & $\mathrm{CL} / \mathrm{P}$ & 1.1 & $3.7 \times 10^{-2}$ & РОМТ1, РОМТ2 \\
\hline & 14 & MTR related & $\mathrm{CL} / \mathrm{P}$ & 1.1 & $4.6 \times 10^{-2}$ & MTHFR, MTR \\
\hline & 15 & Tropomyosin & $\mathrm{CL} / \mathrm{P}$ & 1.1 & $4.6 \times 10^{-2}$ & MYH9*, TMP2 \\
\hline \multirow[t]{2}{*}{ CPO only } & 16 & Sox transcription factors & $\mathrm{CPO}$ & 1.7 & $1.6 \times 10^{-2}$ & SOX2, SOX9, SRY \\
\hline & 17 & Origin of replication binding protein & $\mathrm{CPO}$ & 1.2 & $1.9 \times 10^{-2}$ & CDC6, ORC1 \\
\hline ICP only & 18 & TGF- $\beta$ receptor type I and II & $\mathrm{ICP}$ & 3.3 & $4.0 \times 10^{-2}$ & TGFBR1, TGFBR2 \\
\hline
\end{tabular}

CL/P, cleft lip and/or palate; CPO, cleft palate only; ICP, incomplete cleft palate; SCP, submucous cleft palate; TGF, transforming growth factor; FGF, fibroblast growth factor; MTR, Methyltetrahydrofolate-homocysteine methyltransferase; \%: involved genes/total genes $\times 100 ; P$-value, probabilities were adjusted for multiple comparisons across all PANTHER molecular functions using Bonferroni correction.

${ }^{*}$ Genes associated with nonsyndromic oral clefts.

\section{DISCUSSION}

Palatogenesis involves many diverse genes in a complex process. Oral cleft phenotypes develop when this process is disrupted in some manner because of gene dysfunction. However, oral cleft phenotypes can vary significantly, and this phenotypic variation likely reflects the involvement of different genes and/or changes in the functional contributions of the same genes. To understand better the genetic contributions underlying different oral cleft phenotypes, it is necessary to identify and characterize these culprit genes. It is known that the empirical recurrence risks for $\mathrm{CP}$ and $\mathrm{CL} / \mathrm{P}$ are independent, characterized by differences in sex ratios and prevalence. ${ }^{3}$ Similarly, our ontology analysis found different gene profiles indicating different underlying genetic etiologies of $\mathrm{CP}$ and $\mathrm{CL} / \mathrm{P}$. When genes were analyzed according to molecular function, biological process, chemical-gene-disease interactions, and gene family, we found distinct genetic profiles for different cleft palate phenotypes such as $\mathrm{CL} / \mathrm{P}, \mathrm{CP}$, incomplete $\mathrm{CP}$, and submucous $\mathrm{CP}$. The results of our gene ontology analyses support the findings of earlier epidemiological studies that suggest that different genetic etiologies underlie different oral cleft phenotypes. They further demonstrate the usefulness of ontological candidate gene analysis in understanding gene function in palatogenesis.

Using ontology analysis, we found that the T-box protein family, the collagen- $\alpha$ chain protein family, and the TGF- $\beta$ family were associated with $\mathrm{CPO}$ and incomplete $\mathrm{CP}$. Consistent with our findings, a study reported that TGF- $\beta$ regulates collagen synthesis and degradation, thereby affecting the amount of collagen present in the mesenchyme of the embryonic palate. ${ }^{19}$ The T-box gene, TBX1, is the major candidate gene for DiGeorge syndrome (OMIM \#188400) and may be responsible for several phenotypes including cleft palate, while mutations in TBX22 cause a form of X-linked cleft palate (OMIM \#303400). Similarly, mutations in the collagen-a chain genes, COL2A1, COL9A2, COL11A1, and COL11A2, have been associated with different forms of Stickler syndrome (OMIM\#108300, \#614284, $\# 604841$, and \#184840, respectively), a clinically variable condition that includes cleft palate. As disruption of T-box proteins and collagen-a chain proteins both contribute to $\mathrm{CPO}$ and incomplete $\mathrm{CP}$ in humans, and that $T b x 1$ knockout mice exhibit different $\mathrm{CP}$ phenotypes including incomplete $\mathrm{CP}$ and submucosal $\mathrm{CP},{ }^{20}$ further investigations to determine whether deletion of $T b \times 1$ or $T b \times 22$ affects expression of collagen-a chain genes in mouse palatal shelves are warranted.

In summary, we identified a pool of candidate genes associated with different oral cleft phenotypes. Our gene ontology analysis revealed that genes associated with each cleft palate phenotype show different functional profiles. It is possible that some of the candidate genes identified are involved in tongue or bone anomalies and induce oral clefts during palatogenesis as a secondary defect. In addition, some polymorphisms identified in listed genes may not be disease-causing per se, but benign sequence variants in linkage disequilibrium with 
pathogenic variants. In addition to gene mutations, epigenetic changes and microRNA regulation may alter gene expression during palatogenesis. Nevertheless, the results of the gene ontology analysis indicated distinct genetic profiles for each oral cleft phenotype and differences in the underlying genetic etiologies of oral clefts. Analysis of the candidate genes and their products may provide an opportunity to discover new disease-causing genes implicated in palatogenesis.

\section{ACKNOWLEDGEMENTS}

This work is dedicated to the memory of Dr Kimie Ohyama, a professor emerita of Orthodontics. We thank Editage for their support in editing the manuscript for English language. This work was supported by the Japan Society for the Promotion of Science (JSPS) KAKENHI (grant numbers 25670774, $15 \mathrm{~K} 11004)$ to NF.

1 Mossey PA, Little J, Munger RG et al. Cleft lip and palate. Lancet 2009; 374(9703): 1773-1785.

2 Dixon MJ, Marazita ML, Beaty TH et al. Cleft lip and palate: understanding genetic and environmental influences. Nat Rev Genet 2011; 12(3): 167-178.

3 Fraser FC. Review: the genetics of cleft lip and cleft palate. Am J Hum Genet 1970; 22 336-352.

4 Herkrath AP, Herkrath FJ, Rebelo MA et al. Parental age as a risk factor for nonsyndromic oral clefts: a meta-analysis. J Dent 2012; 40(1): 3-14.

5 Little J, Bryan E. Congenital anomalies in twins. Semin Perinatol 1986; 10(1): 50-64.

6 Rank BK, Thomson JA. Cleft lip and palate in Tasmania. Med J Aust 1960; 47(2): 681-689.

7 Cohen MM Jr.. Malformations of the craniofacial region: evolutionary, embryonic, genetic, and clinical perspectives. Am J Med Genet 2002; 115(4): 245-268.

8 Gritli-Linde A. Molecular control of secondary palate development. Dev Biol 2007; 301 (2): 309-326.
9 Funato N, Nakamura M, Yanagisawa H. Molecular basis of cleft palates in mice. World J Biol Chem 2015; 6(3): 121-138.

10 Jimenez-Sanchez G, Childs B, Valle D. Human disease genes. Nature 2001; 409 (6288): 853-855.

11 Hamosh A, Scott AF, Amberger JS et al. Online Mendelian Inheritance in Man (OMIM), a knowledgebase of human genes and genetic disorders. Nucleic Acids Res 2005; 33 (database issue): D514-D517.

$12 \mathrm{Mi} \mathrm{H}$, Muruganujan A, Casagrande JT et al. Large-scale gene function analysis with the PANTHER classification system. Nat Protoc 2013; 8(8): 1551-1566.

13 Thomas PD, Campbell MJ, Kejariwal A et al. PANTHER: a library of protein families and subfamilies indexed by function. Genome Res 2003; 13(9): 2129-2141.

14 Davis AP, Grondin CJ, Lennon-Hopkins K et al. The Comparative Toxicogenomics Database's 10th year anniversary: update 2015. Nucleic Acids Res 2015; 43(database issue): D914-D920.

15 Benjamini Y, Yekutieli D. The control of the false discovery rate in multiple testing under dependency. Ann Statist 2001; 29(4): 1165-1188.

16 Frech $\mathrm{C}$, Chen N. Genome-wide comparative gene family classification. PLoS One 2010; 5(10): e13409.

17 Depew MJ, Lufkin T, Rubenstein JL. Specification of jaw subdivisions by Dlx genes. Science 2002; 298(5592): 381-385.

18 Funato $\mathrm{N}$, Kokubo $\mathrm{H}$, Nakamura $\mathrm{M}$ et al. Specification of jaw identity by the Hand2 transcription factor. Sci Rep 2016; 6: 28405

19 D'Angelo M, Chen JM, Ugen $\mathrm{K}$ et al. TGF $\beta 1$ regulation of collagen metabolism by embryonic palate mesenchymal cells. J Exp Zool 1994; 270(2): 189-201.

20 Funato N, Nakamura M, Richardson JA et al. Tbx1 regulates oral epithelial adhesion and palatal development. Hum Mol Genet 2012; 21(11): 2524-2537.

(c) (i) This work is licensed under a Creative Commons Attribution 4.0 International License. The images or other third party material in this article are included in the article's Creative Commons license, unless indicated otherwise in the credit line; if the material is not included under the Creative Commons license, users will need to obtain permission from the license holder to reproduce the material. To view a copy of this license, visit http://creativecommons. org/licenses/by/4.0/

(C) The Author(s) 2017

Supplementary Information for this article can be found on the International Journal of Oral Science website (http://www.nature.com/ijos) 